\title{
STUDY AND APPLICATION ON CLOUD COVERED RATE FOR AGROCLIMATICAL DISTRIBUTION USING IN GUANGXI BASED ON MODIS DATA
}

Xin Yang ${ }^{1,2^{*}}$, Shiquan Zhong ${ }^{1,2}$, Han Sun ${ }^{1,2}$, Zongkun Tan ${ }^{1,2}$, Zheng $\mathrm{Li}^{1,2}$, Meihua Ding ${ }^{1,2}$

${ }^{1}$ Remote Sensing Application and Test Base of National Satellite Meteorology Centre, Nanning, China, 530022

${ }^{2}$ GuangXi Institute of Meteorology, Nanning, China 530022

* Corresponding author, Address: GuangXi Institute of Meteorology, Nanning, 530022, P. R. China, Tel::86-771-5875207,Fax:+86-771-5865594,Email:phonenumber@163.com

Abstract: $\quad$ Based on analyzing of the physical characteristics of cloud and importance of cloud in agricultural production and national economy, cloud is a very important climatic resources such as temperature, precipitation and solar radiation. Cloud plays a very important role in agricultural climate division .This paper analyzes methods of cloud detection based on MODIS data in China and Abroad. The results suggest that Quanjun He method is suitable to detect cloud in Guangxi. State chart of cloud cover in Guangxi is imaged by using Quanjun He method.We find out the approach of calculating cloud covered rate by using the frequency spectrum analysis. At last, the Guangxi is obtained. Taking Rongxian County Guangxi as an example, this article analyze the preliminary application of cloud covered rate in distribution of Rong Shaddock pomelo . Analysis results indicate that cloud covered rate is closely related to quality of Rong Shaddock pomelo.

Keywords: MODIS, cloud covered rate, Rong Shaddock pomelo, distribution

Please use the following format when citing this chapter:

Yang, X., Zhong, S., Sun, H., Tan, Z., Li, Z. and Ding, M., 2009, in IFIP International Federation for Information Processing, Volume 293, Computer and Computing Technologies in Agriculture II, Volume 1, eds. D. Li, Z. Chunjiang, (Boston: Springer), pp. 275-284. 


\section{INTRODUCTION}

A cloud is a visible aggregate of tiny water droplets and/or ice crystals suspended in the atmosphere and can exist in a variety of shapes and sizes. Some clouds are accompanied by precipitation; rain, snow, hail, sleet, even freezing rain. Clouds are the maternal body of natural precipitation and precipitation enhancement, the main natural factors influencing moisture supply and water regulation of upland crops, the main regulator of the underlying surface solar radiation. Clouds have specially significant effect on the energy balance of the earth atmosphere. The radiative forcings by cloud is recognized as the one important contributors for climate change. Clouds are closely related with people's daily lives and manufacture .In agricultural production, different cloud layer and cloud cover time have also affected different in different season or different period of time . In a critical time of spring sowing, moisture in the fields was evaporated in warm and fine weather, spring drought may cause and have an bad effect; Clouds could effectively screen out much of the sun's radiation in the summer, it can prevent the seedling of crops and fruits of Shatian pomelo, oranges ,etc. from injury of the sun's radiation. In winter, two reasons may cause freeze injury .one is longer cloud cover time in the daytime, the other is longer clear time at night. Land have not enough solar radiation to absorb energy because of cloud cover in day; freeze injury hurt crops especially warmtemperate crops.

Clouds are vital significance to the national economy .It is not only in plant diseases and insect pests of agricultural production but also in safety of civil aviation or on water or air. Ground proximity clouds may generate Fogs under some situations. Fogs have become the first largest killer on highway. An airplane can't take off on schedule because of the heavy fog.

Clouds are determined by main physical factor of climate formation. Cloud cover changes the balance of underlying surface earth-atmosphere radiation, leading to the surface energy and moisture budgets of earthatmosphere system. To some extent, cloud is a very important climatic resources such as temperature, precipitation and solar radiation. It is helpful to understand characteristics of cloud .

A completely clear day might seem boring in a cloud observer project, but in fact this is one of the most important things a surface observer can report. Clear sky is easy to detect from the ground, because one observes against the uniform background of space. When looking down at the Earth, however, the background is highly variable and can make cloud detection very hard. In conventional meteorological observation, cloudiness estimation is using by visual observations of agro-meteorological observer. The method is :The sky 
is divided into 10 equal divisions, cloud covered divisions are regarded as cloudiness .

Clear (cloudiness $<3 / 10$ )

Cloudy (cloudiness $3 / 10$ to $8 / 10$ )

Overcast (cloudiness $>9 / 10$ )

Using this method to estimate cloudiness, the result represents only observation station. Conventional meteorological observation can't accurately reflect the change of cloudiness of local area and beyond observation. With the development of satellite remote sensing technology, meteorological satellites and weather radar are using to monitor cloudiness . According to analysis theory and present Situation of cloud detection, this paper mainly studies on obtain cloud coverage rate image of Guangxi Province based on MODIS(Moderate Resolution Imaging Spectroradiometer) data. The objective is to make better use of cloud resources to serve the society.

\section{CLOUD DETECTION METHODS}

\subsection{Basic principle and research status of cloud detection}

Clouds are generally characterized by higher reflectance and lower temperature than the underlying earth surface. As such, simple visible and infrared window threshold approaches offer considerable skill in cloud detection. However, there are many surface conditions when this characterization of clouds is inappropriate, most notably over snow and ice. Additionally, some cloud types such as thin cirrus, low stratus at night, and small cumulus are difficult to detect because of insufficient contrast with the surface radiance. Cloud edges cause further difficulty since the instrument field of view will not always be completely cloudy or clear.

MODIS offers the opportunity for multispectral approaches to cloud detection so that many of these concerns can be mitigated; additionally, spatial and temporal uniformity tests offer confirmation of cloudy or clearsky conditions.

There are many MODIS cloud algorithms :

Menzel et at., use infrared band to detect cloud(Menzel,1993), the six concrete judgment basis as follows: TB11 $+\mathrm{a}(\mathrm{PW}) \times \mathrm{DTB}(11,12), \mathrm{TB} 11+$ b(PW )×DTB $(11,8.6)$, TB11 +C(PW ),DTB $(4,11)$, TB13, 1.38 , whrer a,b,C is respectively function of $\mathrm{PW}$. if one of six judgment basis more than 
specific threshold shows that this pixel is covered by cloud, if none of six judgment basis more than specific threshold shows that this pixel is clear.

The algorithm offered by MODIS cloud mask team is widely used in the world at present. This algorithm is determined for good data only (i.e., fields of view where data in MODIS bands 1, 2, 4, 5, 6, 7, 17, 18, 19, 20, 22, 26, $27,28,29,31,32,33$, and 35 have radiometric integrity). Incomplete or bad radiometric data creates holes in the cloud detection. Additionally, the cloud mask requires several ancillary data inputs such as sun angle, azimuthal angle, topography, ecosystems, Daily NISE snow/ice map and Daily sea ice.The algorithm is easily influenced by working condition of detector.

In China, an automatic cloud detection algorithm is applied based on the spatial texture analysis and neural network(Song, 2003), judgment basis as follows: $\rho 0.66, \mathrm{~TB} 12, \rho 0.87 / \rho 0.66, \mathrm{INDV}=(\rho 0.87-\rho 0.66) /(\rho 0.87+$ $\rho 0.66)$,DTB $(3.7,11)$, DTB $(8.6,11)$, ICD: $\quad(\rho 0.66-\rho 0.936) /(\rho 0.66+$ $\rho 0.936$ ) . Xia Sheng puts forward a new method to discriminate cloud from clear scene utilizing M ODIS visible and infrared bands data, which proved the efficiency of the method in mid or low latitude regions with vegetation or ocean background(Sheng,2004).Through analyzing meteoric characteristics of cloud in different spectra and integrating the spectral characteristics of MODIS, the authors(He,2006) put forward a new cloud detection method based on multi-spectrum synthesis.

\subsection{Cloud detection method}

Based on the characters of semi-tropical climate,topography,vegetation in Guangxi, the methods for cloud detection in MODIS data are analyzed and compared . The results suggest that the method offered by Quanjun $\mathrm{He}$ is suitable to detect cloud in Guangxi. The Human Computer Interaction Platform based on the slip threshold figure is used in our cloud detection method.

The MODIS bands used in the cloud detection method are identified in Table 1

Table 1. MODIS bands used in the MODIS cloud detection method

\begin{tabular}{lll}
\hline Band & Wavelength $(\mu \mathrm{m})$ & Comment \\
\hline CH1 & $0.620 \sim 0.670$ & clouds, shadow \\
CH6 & $1.628 \sim 1.652$ & snow, shadow \\
CH8 & $0.405 \sim 0.420$ & ocean \\
CH26 & $1.360 \sim 1.390$ & thin cirrus, high cloud \\
CH29 & $8.400 \sim 8.700$ & Brightness temperature \\
& & difference \\
CH31 & $10.780 \sim 11.280$ & window threshold \\
CH31 & $10.780 \sim 11.280$ & window threshold \\
\hline
\end{tabular}

(concrete method can be seen Quanjun He et at., 2006) 


\section{OBTAIN CLOUD COVERAGE INDEX IMAGE OF GUANGXI PROVINCE}

\subsection{Study area and data source}

The study area is located in Guangxi province, south of China. It's latitude is $20^{\circ} 54^{\prime} \sim 26^{\circ} 23^{\prime} \mathrm{N}$ and longitude is $104^{\circ} 29^{\prime} \sim 112^{\circ} 04^{\prime} \mathrm{E}$. Its total area is $236700.0 \mathrm{~km}^{2}$. It belongs to monsoon region of south subtropical zone and north tropical zone without four clearly demarcated seasons of spring, summer, autumn and winter. The climate here is hot and humid in summer and warm and dry in winter.

Data input to the cloud detection method is assumed to be calibrated and navigated level 1B radiance data which offered by National Satellite Meteorological Center and DVBS of GuangXi Institute of Meteorology. The time segment of complete data is from 2001 to 2006.

\subsection{Cloud covered rate image in Guangxi}

The detailed work and the corresponding results are as follows:

(1)Useing Quanjun He method to detect cloud of all MODIS data, We assumption that:

$$
R_{i, j}= \begin{cases}1, & \text { cloud pixel } \\ 0, & \text { no-cloud pixel }\end{cases}
$$

Where: $R_{i j}$ is pixel of MODIS image.

State chart of cloud cover is obtained based on cloud detection of $R_{i j}$.

(2) Calculating cloud covered rate by employing templates

Suppose size of image $\mathrm{R}$ is $\mathrm{m} \times \mathrm{n}$, we employ templates as follows :

$$
\begin{aligned}
& \mathrm{P}=\left\{\mathrm{R}_{\mathrm{ij}}\right\}_{\mathrm{m} \times \mathrm{n}} \quad \mathrm{i}, \mathrm{j}=1,2, \ldots \mathrm{p} \\
& \mathrm{Q}_{\mathrm{p}}=\left\{\mathrm{q}_{\mathrm{f}, \mathrm{g}, \mathrm{p}}\right\}_{\mathrm{m}-\mathrm{p}+1, \mathrm{n}-\mathrm{p}+1, \min (\mathrm{m}, \mathrm{n})-1}
\end{aligned}
$$

Where: $\mathrm{P}$ is gliding template, $\mathrm{Q}_{\mathrm{p}}$ is spectrum statistical Matrix

For all sample graph, use formula (2) to investigate region of cloud covered. If $\mathrm{i}=1$ and $\mathrm{j}=1$ in gliding template $\mathrm{P}$, corresponding position of image $\mathrm{R}$ is $\mathrm{f}_{1}, \mathrm{~g}_{1}$, then corresponding point in $\mathrm{Q}_{\mathrm{p}}$ is point $\mathrm{f}_{1}, \mathrm{~g}_{1}, \mathrm{p}$.According to formula (1),if $\left\{\mathrm{R}_{\mathrm{ij}}\right\}=1$, that is template covered by cloud, The count of $\mathrm{Q}_{\mathrm{p}}$ add $\mathrm{s}$ one, otherwise, the count of $\mathrm{Q}_{\mathrm{p}}$ keeps invariant.

Using above methods, we obtain cloud covered rate image of Guangxi by counting all MODIS data(Fig.1- Fig.5) . 


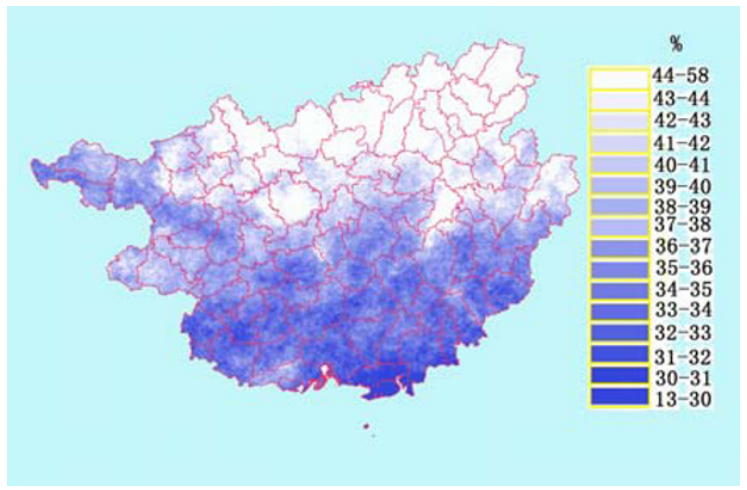

Fig. 1: The annual-averaged cloud covered rate image of Guangxi

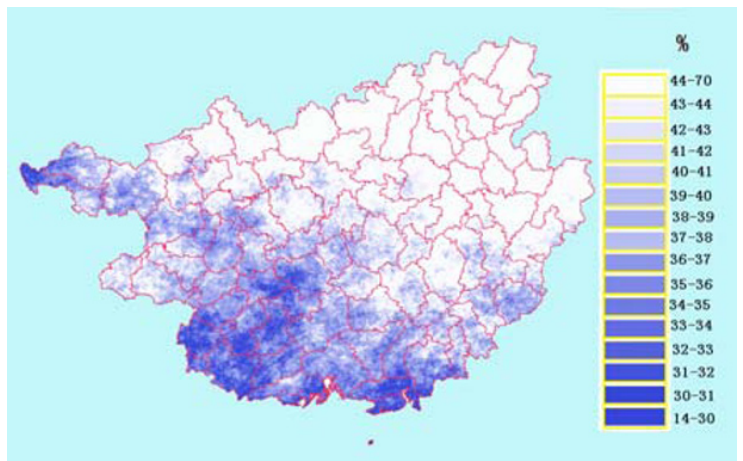

Fig.2: The spring- averaged cloud covered rate image of Guangxi

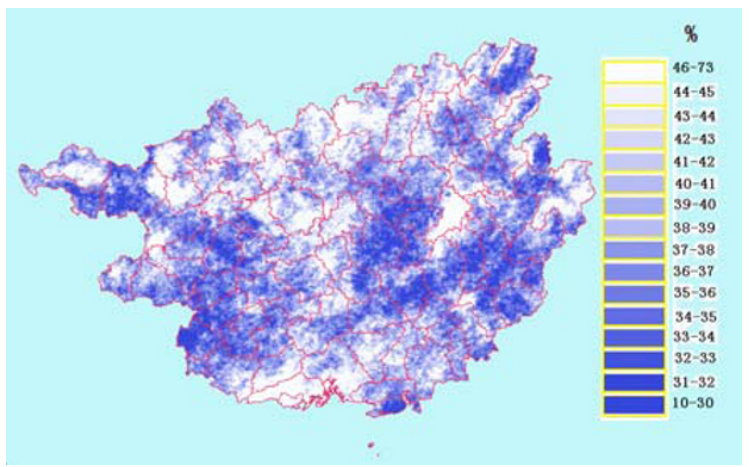

Fig.3: The summer- averaged cloud covered rate image of Guangxi 


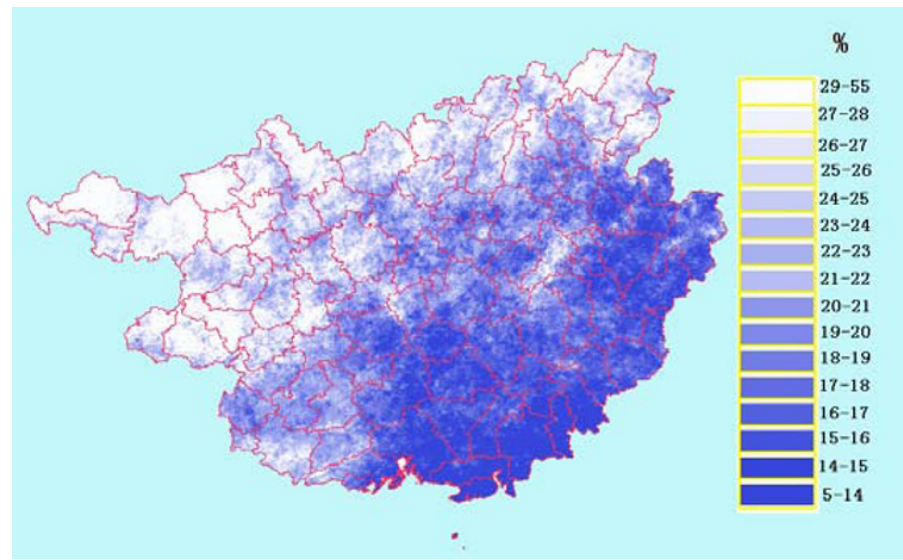

Fig.4: The autumn- averaged cloud covered rate image of Guangxi

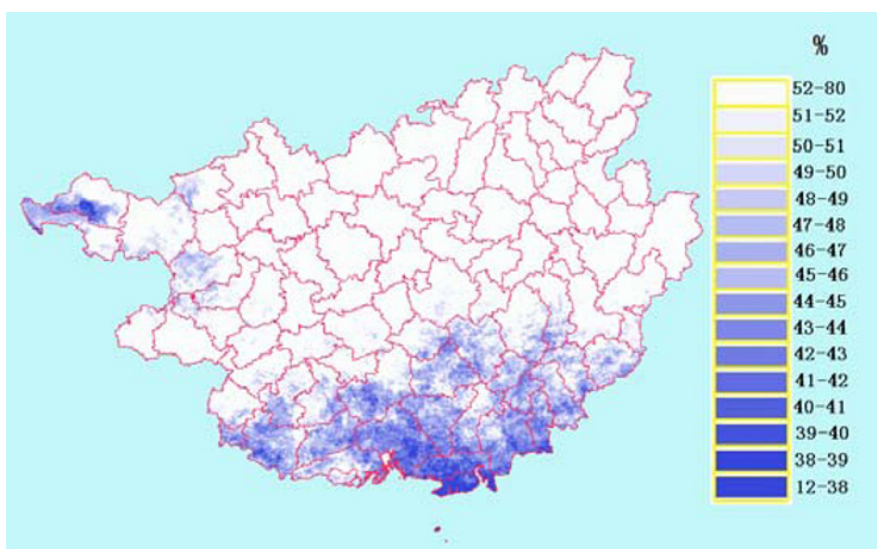

Fig.5: The winter- averaged cloud covered rate image of Guangxi

\subsection{Preliminary application of cloud covered rate in distribution of Rong Shaddock pomelo}

Shaddock pomelo is a treasure of Chinese pummel, and is primary export fruit,mainly distributed in some areas of Guangxi Province Guangdong Province Sichuan Province and Hunan Province. During the last decade, along with the rapid development of Shaddock pomelo production, many problems have appeared such as taste of fruit reduced, economic benefit drops and low unit yield. (Su,2005)According to the requirement of Shatian pomelo cultivar to climatic conditions, and based on the analysis of the climatic data of 90 weather stations accumulated in 30 


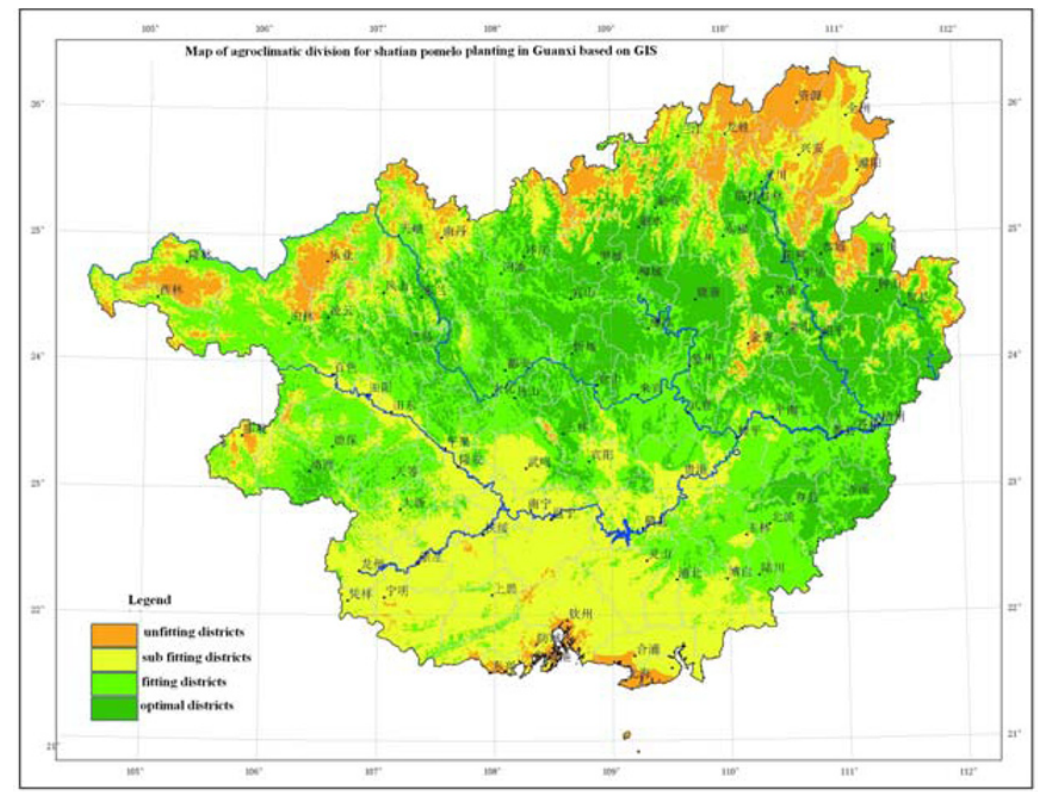

Fig.6: Map of agroclimatic division for shatian pomelo planting in Guanxi based on GIS

based on GIS years and the fundamental geographic data on the scale of 1 to 250000 in Guangxi,the optimal,fitting,sub fitting and unfitting districts for growing Shatian pomelo cultivar are regionalized(Fig.6) .

Rongxian County is one of high quality producing areas in Guangxi, we take Rongxian County as an example to analyze the preliminary application of cloud covered rate in distribution of Rong Shaddock pomelo .

Fig.7 show that A is the optimal district in Rongxian County, B is the fitting district.In Fig.1, cloud covered rate is relatively low in the central and northern regions of Rongxian County, A have comparatively high absorption for Solar Radiation, so the annual-averaged temperature is comparatively high .It is helpful to growth of Shaddock pomelo. B have comparatively low absorption for Solar Radiation, It is not benefit to growth of Shaddock pomelo. Shaddock pomelo starts flowering in April. Good weather is benefit to flowering. Cloud covered rate of $\mathrm{A}$ is lower than that of B (Fig.2),so A has ensured a good flowering rate. In the growing season, cloud covered rate of $\mathrm{B}$ is higher than that of A (Fig.3), high cloud covered rate may lead to abnormal weather. It is not helpful to fruit set percentage of Shaddock pomelo. The fruit maturation period is in the last ten days of October, enough light radiation is favorable to the sugar accumulation and flavor keeping in fruits(Fig.4). Suitable light radiation is 
benefit to Aromatic Mixture, so Shaddock pomelo has a strong aroma. But strongly light radiation may cause heat injury to fruit of Shaddock pomelo.
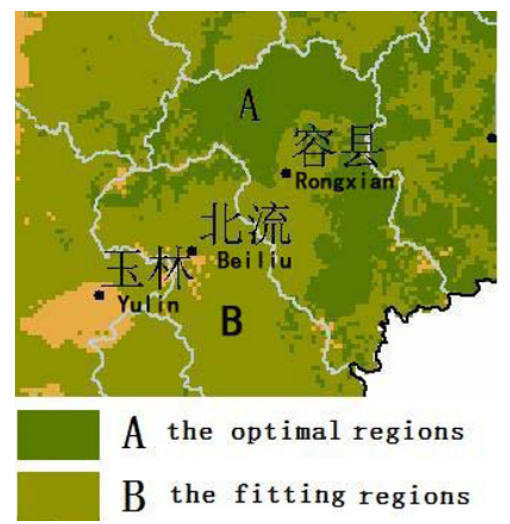

Fig.7: Map of shatian pomelo planting in Rongxian County Guanxi

\section{CONCLUSION}

(1)Cloud covered rate was first used for agricultural climate division in this paper. Analysis results indicate that cloud covered rate is closely related to quality of Rong Shaddock pomelo. Cloud is a very important climatic resources such as temperature, precipitation and solar radiation. It is helpful to agricultural climate division .

(2)Calculation method of cloud covered rate image is given in this paper.

(3)Period of MODIS data is relatively less, it has certain effect to agricultural climate division .But it does not affect methods utility. With the development of satellite remote sensing technology, questions will been solved.

\section{ACKNOWLEDGEMENTS}

This research was supported by National 11th Five-Year Plan major scientific and technological issues (2006BAD04B03) and National Key Technologies R\&D Program (2008BAD08B01), It is also supported by the Guangxi Meteorological Bureau new technology extend project

(QK200602),Sincerely thanks are also due to Guangxi Climate center and National Satellite Meteorology Center for providing the data for this study. 


\section{REFERENCES}

ACKERMAN S.STRABALA K.MENZEL P.Discriminating clear-sky from cloud with MODIS algorithm theoretical basis document (MOD35)[EB/OL].ftp://eospso.gsfc.nasa. gov/ATBD/REVIEW/MODIS/ATBD-MOD-35/atbd-mod-35. pdf,1997-11-01

BAUM,TREPTE Q.A grouped threshold approach for scene identification in AVHRR imagery[J]. Journal of Atmospheric and Oceanic Technology,1999(16):793-799

DERRIENM,LE G H. Cloud classification extracted from AVHRR and GOES imagery[R],Pairs: MeteoFrance SCEM Centre de Meteorologic Spatiale,1998

GRIFFIN M,BURKE H,MANDL D.Cloud cover detection algorithm for EO-1 hyperion imagery[A].In:EO-1 SVT Meeting[C].Hilo: MIT Lincoln Laboratory NASA.2002

Hongmei Zhou, Jianguo Tan, Weiqiang Ge. Automatic detection and rehabilitation of cloud / fog pollution in NOAA/AVHRR imagery. Journal of Natural Disasters,2003,12(3): 4147

Hongmei Zhou, Xingwei Yang, Xian Lu. Study on Detecting Cloud of NOAA Satellite Image. Remote Sensing of Environment China,1995,10(2): 13-142

JEDLOVEC G J. LAWS K. Operational cloud detection in GOES imagery[A].In:11th conference on Satellite Meteorology and Oceanography[C].Madison: Univ of WI,Madison,2001

MENZEL P.Investigation of cloud properties and atmospheric stability with MODIS[R]. Madison:NOAA/NESDIS at the University of W isconsin, 1993

Quanjun He, Jing Cao,Jiang Huang,et al. Cloud Detection in MODIS data based on multispectrum synthesis. Remote Sensing for Land \& Resources,2006,3:19-21

Xia Sheng, Longxiang Sun, Qingmei Zheng. Cloud Detection Using MODIS Data. Journal of PLA University of Science and Technology,2004,5(4):98-101

Xiaoning Song, Yingshi Zhao. Cloud Detection and Analysis of MODIS Image [J]. Journal of Image and Graphics,2003,8(9):1079-1083

Yongxiu Su,Zheng Li,Meihua Din, et al. Study on the agro-climatic regionalism for growing Shatian pomelo cultivar in Guangxi Autonomous Region by GIS technology,2005,22(5), 500-504 